\title{
Mechanical, but not infective, pacemaker erosion may be successfully managed by re-implantation of pacemakers
}

\author{
Michael J Griffith, J Paul Mounsey, Rodney S Bexton, Michael P Holden
}

\begin{abstract}
Objective-When a pacemaker box causes erosion it is usually removed and a new pacemaker implanted at a contralateral site. In this study when there was no evidence of systemic infection an attempt was made to clean and reimplant the same pacemaker in the same site. Results-Over 10 years 62 patients had pacemaker reimplantation. In 18 patients the procedure was repeated a second time. Reimplantation was successful after at least six months follow up in 38 patients (61\%): in nine two attempts had been made. Mean hospital stay for all patients was 21.3 days; for patients in whom the procedure was successful it was 12.5 days and for those in whom it was unsuccessful it was 35.4 days. $31(82 \%)$ of the 38 patients in whom reimplantation was successful had no bacterial growth from wound swabs. Bacteria were cultured from wound swabs from $17 / 24(71 \%)$ patients in whom reimplantation was unsuccessful $(p<0.001)$. Bacteria were grown from swabs from $7 / 8$ patients with a protruding wire compared with $9 / 23$ patients with a protruding pacemaker $(p=0.05)$. Thin patients and those who were older were more likely to have successful reimplantation: neither association reached statistical significance. A clinical impression of infection was not helpful. If re-implantation had been attempted only in the patients with negative wound swabs or intact skin the success rate would have been $74 \%$ at a cost of $£ 5010$ per patient compared with a cost of $£ 6509$ per patient for explantation and a reimplantation of a new contralateral pacemaker.

Conclusion-These data support the hypothesis that pacemaker erosion is caused by primary infection or by a noninfective process (probably mechanical pressure). Pacemaker erosion that is not caused by infection can be successfully managed by ipsilateral reimplantation and this approach saves money.
\end{abstract}

(Br Heart f 1994;71:202-205)

Implanted permanent pacemakers cause erosion of infection in $0.13 \%$ to $12 \cdot 6 \%$ of patients. ${ }^{1}$ In the early 1970 s there were reports of successful conservative management with closed antibiotic irrigation. ${ }^{23}$ More recently the problem of pacemaker erosion has been assumed in many centres to be caused by infection and is managed by explanting the pacemaker and implanting a new pacemaker on the contralateral side. ${ }^{4-7}$ At our centre the first line of treatment has been to reposition the same pacemaker, which is cleaned and usually implanted in a new pocket on the same side as the previous implant. This paper assesses the long-term results of this strategy and attempts to identify a patient subset in whom reimplantation is particularly successful.

\section{Patients and methods}

PATIENTS

We studied 62 consecutive patients who had had their pacemaker repositioned between 1981 and 1991 by one surgeon. There were 2949 new pacemakers implanted in this period. Most patients who presented with an infected, painful, or eroded pacemaker were referred for repositioning of the pacemaker except when there was gross local sepsis or septicaemia. Forty seven patients were treated with immediate implantation of a contralateral pacemaker without initial repositioning. Thus a total of 109 patients (3.7\%) had problems, of whom 71 had new pacemakers implanted $(2 \cdot 4 \%)$. The same pocket was used in 37 patients and a new pocket was made in 25 patients. The mean age of the patients at the first implantation was $62 \cdot 8$ (range $24-88$ ) years, and 27 were women. Twenty one patients had DDD units implanted, two patients had AAI units, and 39 patients had VVI units. One patient had an epicardialepigastric system and the sites in the others were endocardial and prepectoral. The original surgeon was of consultant status in 26 patients. Antibiotic prophylaxis was given at the original implant in 33 patients. At presentation three patients had pain with no tenderness around the site of the pacemaker, 21 patients had discoloured but unbroken skin, 23 had erosion of the pacemaker box, eight patients had erosion of the wire, five patients had the pacemaker completely out on the skin surface, and two had tender pacing sites with no other changes. The clinical impression was 
that 35 patients had signs of infection. In 38 patients no bacteria could be cultured. Staphylococcus aureus was grown from nine patients, Staphylococcus epidermidis from four patients, haemolytic streptococcus from one patient, and skin flora from 10 patients. Five patients were diabetic.

\section{SURGICAL PROCEDURE}

The reimplantation procedure was carried out under general anaesthesia. Wound swabs were taken from the exposed site. Intravenous antibiotic cover, flucloxacillin in the absence of allergy, was given during and after the procedure. The antibiotics were adjusted if indicated when the information from wound swabs became available. Granulation tissue was excised and, where appropriate, a new bigger pocket was fashioned ( 15 patients) or a new ipsilateral site was used (eight patients). In patients where the same pocket was used the floor of the pocket was incised and the pocket was expanded in a medial and caudal direction in the immediate prepectoral plane. All foreign bodies, such as black silk, were excised. The pacemaker box and exposed wire were cleaned with iodine. Generally the pacemaker box was not disconnected from the pacing wire or wires. If the patient was dependent on pacing, electrical contact was maintained between the box and patient during cleaning. Deep tissues were closed and the skin sutured with absorbable synthetic sutures. The antibiotics were continued for 5 days or more after operation. The days in hospital were recorded for the periods related to repositioning. In 18 patients the procedure was repeated a second time, but never more than this.

\section{FOLLOW UP}

Patients were followed up in the pacemaker clinic every six months.

\section{END POINTS}

A successful result was defined by a clean non-tender pacemaker site at follow up of at least six months. Failure was defined as the need for explantation of the pacemaker with a new pacemaker implanted on the contralateral site. The pacemaker was explanted if infection was apparent soon after the first attempt at reimplantation or if infection or erosion occurred after the second reimplantation.

Table 1 Factors predicting the long-term success of pacemaker reimplantation

\begin{tabular}{llll}
\hline Factor & $\begin{array}{l}\text { Successful } \\
\text { reimplantation } \\
(n=38)\end{array}$ & $\begin{array}{l}\text { Explantation } \\
(n=24)\end{array}$ & $p$ Value \\
\hline Unbroken skin & 17 & 7 & $0 \cdot 22$ \\
No bacterial growth & 31 & 7 & $0 \cdot 001$ \\
Antibiotics & 23 & 10 & $0 \cdot 147$ \\
DDD & 14 & 7 & $0 \cdot 53$ \\
Thin & 11 & 3 & $0 \cdot 13$ \\
Clinical & 23 & 12 & $0 \cdot 42$ \\
infection & $64 \cdot 9(15)$ & $59 \cdot 5(15)$ & $0 \cdot 17$ \\
Age (SD, yr) & $22 \cdot 4(4)$ & $16(2 \cdot 8)$ & $0 \cdot 26$ \\
Time to & & & \\
reimplantation & & & \\
(SD, months) & & &
\end{tabular}

^Time from original pacemaker implantation to first attempted reimplantation.

\section{FINANCIAL AUDIT}

A bed day was costed at $£ 202$. Theatre time was calculated at $£ 376$ hour and a reimplantation was calculated to take 1.21 hours $(£ 456)$. Pacemaker costs were calculated at 1992 prices, with a VVI pacemaker costing $£ 632$, an AAI pacemaker $£ 927$, and a DDD pacemaker $£ 1811$. Capital charges were added at $9 \%$ and value added tax at $17 \cdot 5 \%$. The alternative strategy of a new pacemaker after explantation of the old unit was costed based on the study published by Choo et al. ${ }^{4}$ The calculations were based on the projected cost of their experience if it had been at our centre in 1992. They performed two operations with interim pacing in 18 patients (mean stay in hospital 27 days with a repeat operation in two patients) and a single operation in 15 patients (mean stay in hospital 19 days). Their single operation involved initial implantation of a new pacemaker on the contralateral side before explantation of the infected pacemaker by a different team. Both of their strategies were assumed to use the equivalent of two theatre sessions.

\section{STATISTICAL ANALYSIS}

The significance of continuous data was determined by Student's $t$ test and that of non-continuous data was by the $\chi^{2}$ test.

\section{Results}

In $38(61 \cdot 3 \%)$ patients pacemakers were successfully reimplanted, nine of these after a second operation. Failure occurred in 24 $(38.7 \%)$ patients who required a new, contralateral pacemaker, nine of these after a second operation. Mean follow up after reimplantation was 21.3 months, with no difference between successful (20.3 months) and failed operations $(22 \cdot 2$ months). There were no deaths related to an infected pacemaker, and septicaemia was seen in six patients. Table 1 shows the influence of various factors in predicting success. The most significant factor was the absence of bacterial growth from wound swabs. Bacteria were cultured from wound swabs taken from seven of the 38 patients who had an ultimately successful reimplantation, compared with positive cultures in 17 of the 24 patients who eventually had their pacemaker explanted $(p<0.001)$. Reimplantation was successful in only $1 / 13$ patients in whom Staphylococcus aureus or Staphylococcus epidermidis was isolated and in $5 / 10$ patients in whom skin flora was grown. Of the five patients who were diabetic, two had a successful reimplantation. Bacteria were cultured in $7 / 8$ patients with a protruding wire (with successful reimplantation in $2 / 8$ of these patients) compared with $9 / 23$ patients in whom the pacemaker was visible, $\mathrm{p}=0.05$ (with successful reimplantation in $14 / 23$ of these patients, $p=0 \cdot 18$ ). Patients who underwent successful reimplantation tended to have unbroken skin over the pacemaker, and to have had antibiotics at the original operation and a longer time from their original implant to reimplantation. They 
Table 2 Length of hospital stay (days) and its relation to outcome

\begin{tabular}{llr}
\hline & Mean (SD) & Range \\
\hline All patients & $21.3(21.9)$ & $2-95$ \\
Success after: & $11 \cdot 8(21 \cdot 9)$ & $2-95$ \\
Primary reimplantation & $14.4(17 \cdot 8)$ & $5-28$ \\
Secondary reimplantation & $12.5(15 \cdot 8)$ & $2-95$ \\
All success & & \\
Failure after: & $37.9(21 \cdot 1)$ & $12-83$ \\
Primary reimplantation & $31.2(27)$ & $6-68$ \\
Secondary reimplantation & $35.4(23 \cdot 1)$ & $6-83$ \\
All failure & 3.4 \\
\hline
\end{tabular}

were also older and thinner but none of these factors reached statistical significance. The clinical impression of infection was not a useful guide to potential success. All five patients with a tender or painful pacing site and no other changes had negative bacterial cultures and their pacemakers were successfully reimplanted. The pacemaker was successfully reimplanted in $31(74 \%)$ of the 42 patients who had either no bacterial growth from wound swabs or unbroken skin. None of the four patients with unbroken skin but positive wound swabs had successful reimplantation.

Table 2 shows the duration of stay in hospital for patients with the various outcomes. It was significantly longer with unsuccessful reimplantation (35.4 days $v 12.5$ days for successful reimplantation).

\section{FINANCIAL AUDIT}

Table 3 shows the costs. The mean cost of reimplantation was $£ 6027 /$ patient, irrespective of success. This is similar to the costs calculated by Choo et al for the single replacement operation $(£ 6509)$ and is much cheaper than their two operation strategy, which cost a mean of $f 8527.4$ Successful reimplantation of the old pacemaker cost a mean of $£ 3348$, which compares favourably with these costs. The mean cost of reimplanting a pacemaker in the 38 patients with negative bacterial cultures was $£ 4492$ /patient, including hardware costs for seven new pacemakers (six VVI), a mean of 1.4 operations, and 16.5 (range 2-95) days in hospital. The mean cost of reimplanting the pacemaker in the 42 patients who either had unbroken skin or negative bacterial cultures was $£ 5010 /$ patient, including 11 new pacemakers (seven VVI), a mean of 1.67 operations, and $17 \cdot 5$ (range 2-95) days in hospital.

Table 3 Cost of pacing strategies per patient

\begin{tabular}{llccc}
\hline & $\begin{array}{l}\text { Hospital } \\
\text { stay }\end{array}$ & Theatre & Hardware & $\begin{array}{c}\text { Total } \\
(+9 \%)\end{array}$ \\
\hline All patients & 4303 & 779 & 461 & 6027 \\
Success after: & & & & \\
$\quad$ Primary reimplantation & 2384 & 456 & - & 3095 \\
$\quad \begin{array}{l}\text { Secondary reimplantation } \\
\text { All success }\end{array}$ & 2909 & 912 & - & 4164 \\
Failure after: & 2525 & 564 & - & 3348 \\
$\quad$ Primary reimplantation & 7656 & 912 & 1183 & 10629 \\
$\quad \begin{array}{l}\text { Secondary reimplantation } \\
\text { All failure }\end{array}$ & 6302 & 1368 & 1204 & 9672 \\
New pacemaker (Choo et al $^{4}$ ): & 7151 & 1083 & 1191 & 10275 \\
2 Stage & 5454 & 1012 & 1357 & 8527 \\
I Stage & 3838 & 912 & 1222 & 6509 \\
\hline
\end{tabular}

\section{Discussion}

The reimplantation of pacemakers was successful in $61 \%$ of patients, $47 \%$ at the first attempt and $14 \%$ at the second. The most significant predictor of success is the absence of bacterial growth from wound swabs. Reimplantation was only successful in $29 \%$ of patients in whom cultures from wound swabs were positive. By contrast patients with primary mechanical erosion (as' determined by the absence of bacterial growth from swabs) were successfully managed by reimplantation with clinical success in $82 \%$ of patients in this study. This finding supports the hypothesis that there are two mechanisms causing local problems to a pacemakernamely, infection and non-infective erosion. Non-infective erosion may be due to mechanical pressure of the pacemaker eroding through the tissues or rejection of a chemical or immunological irritant such as nonabsorbable silk sutures. Mechanical pressure as the cause of non-infective erosion is supported by the trends we found. Successful reimplantation was more likely in older and thinner patients, who have poorer or less tissue. These patients are more likely to have a mechanical cause for their erosion and therefore have a better result achieved with a new, deeper surgical pocket. Infection as a cause of failed reimplantation was supported by the finding that successful reimplantation was less likely in patients who did not receive antibiotics at their original implant. None of these factors, however, reached significance. Erosion of the electrode was much more likely to be related to infection than erosion of the pacemaker box. Eroded electrodes lowered the chance of successful reimplantation of the pacemaker system.

The alternative strategy of removal of the entire system with a new unit implanted on the contralateral side is that most widely practised and has a reported success rate of $>90 \%{ }^{4-7}$ Choo et al were successful in 31/33 patients, either as a one or two stage operation. ${ }^{4}$ Lewis et al were successful in 71/75 patients with a similar one or two operation, which included patients from the previous study. ${ }^{47}$ In these studies the two recurrences of infection were in the group who had two operations. In both of these studies pathological bacteria were grown from wound swabs in all patients, so none of their patients would seem to have had non-infective mechanical erosion. In a study by Harjula et al 43 patients had an attempted reimplantation with recurrence of infection in $33(77 \%)$ patients. ${ }^{6}$ In 25 patients who had the pacemaker totally removed, only two (8\%) infections recurred. Again in this study all patients had bacteria grown from their wounds. Support for conservative treatment for noninfective erosion is found in other reports. In the paper by Kenelly and Piller local surgery was performed on four patients in whom no bacteria were found. Reimplantation was successful in three patients. ${ }^{5}$ It has been suggested that infections with organisms of low virulence may respond to conservative mea- 
Scheme for management of pacemaker erosion.

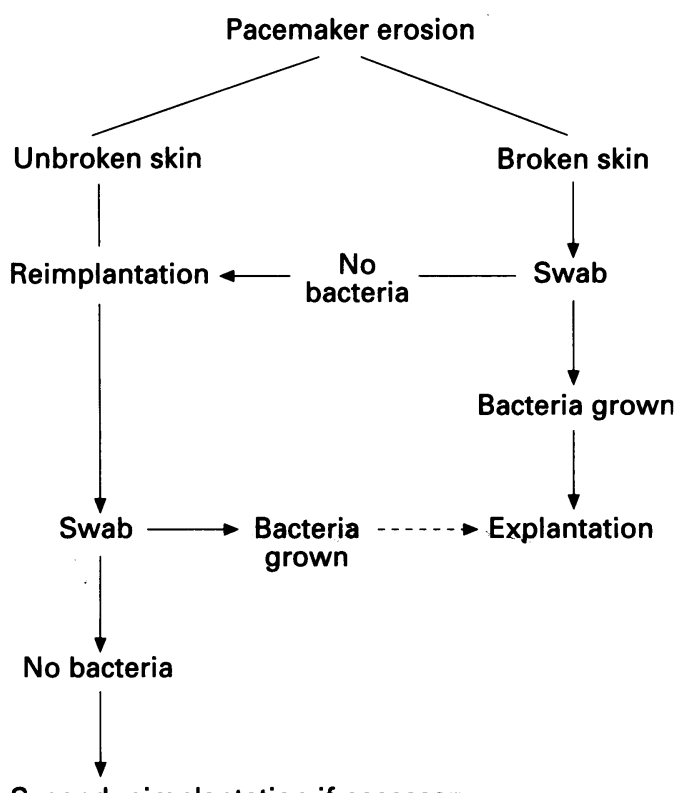

Second reimplantation if necessary

sures. Jara et al found that although Staphylococcus epidermidis was cultured from the infected pacemaker, conservative measures (closed irrigation or surgery) were successful in nine patients. ${ }^{8}$ Follow up times were not reported. In our study none of the four patients with Staphylococcus epidermidis grown from wound swabs had a successful reimplantation.

Financially, attempted reimplantation compared favourably with the alternative strategy of a new contralateral pacemaker based on the figures of Choo et al. ${ }^{4}$ If patients were selected in whom bacterial cultures were negative, then the cost of reimplantation ( 4492$)$ was less than that of an immediate new pacemaker ( $£ 6509)$. This may not be a fair comparison, however, as it is patients with infected pacemakers who probably have the longest stays in hospital, whatever strategy is used. A problem with recommending reimplantation on the basis of microbiological results is that in the absence of either a discharge, frank erosion, or positive blood cultures, wound swabs can only be obtained at the time of surgery. This is too late to alter strategy. A clinical impression of infection was not helpful in our study. If, however, only the 42 patients with either unbroken skin or negative wound swabs had had attempted reimplantation, a $74 \%$ success rate would have been reached at a cost of $£ 5010$. A reasonable strategy would therefore be to proceed to repositioning if the skin was unbroken or if swabs from an open wound were negative. If swabs from the operation were positive then there should be a fairly low threshold for proceeding to explantation (figure).

In conclusion, these data suggest that pacemaker erosion is caused by two different mechanisms-primary infection or a noninfective cause, probably mechanical pressure. Pacemaker erosion that is not caused by infection may be successfully managed by ipsilateral reimplantation, and this strategy is also justified on financial grounds. Infected pacemakers should be explanted.

1 Wade JS, Cobbs CG. Infections in cardiac pacemakers. Curr Clin Top Infect Dis 1988;9:44-61.

2 Golden GT, Lovett WL, Harrah JD, Wellons HA, Nolan SP. The treatment of extruded and infected permanent cardiac pulse generators: application of a technique of closed drainage. Surgery 1973;74:575-9.

3 Furman RW, Hiller AJ, Playforth RH, Bryant LR, Trinkle JK Infected permanent cardiac pacemaker-management without removal. Ann Thorac Surg 1972;14:54-8.

4 Choo MH, Holmes DR, Gersh BJ, et al. Permanent pacemaker infections: characterization and management. $\mathrm{Am}$ f Cardiol 1981;48:559-64.

5 Kennelly BM, Piller LW. Management of infected transvenous permanent pacemakers. Br Heart $\mathcal{F} 1974 ; 36$ : venous per.

6 Harjula A, Jarvinen A, Virtanen KS, Mattila S. Pacemaker infections-treatment with total or partial pacemaker infections-treatment with total or partial pacemaker
system removal. Thorac Cardiovasc Surg 1985;33: system

7 Lewis AB, Hayes DL, Holmes DR, Vliestra RE, Pluth JR, Osborn MJ. Update on infections involving permanen pacemakers. $\mathcal{F}$ Thorac Cardiovasc Surg 1985;89:758-63.

8 Jara FM, Toledo-Pereyra L, Lewis JW, Magilligan DJ. The infected pacemaker pocket. $\mathcal{f}$ Thorac Cardiovas Surg 1979;78:298-300. 